\title{
Deploying Swarm Intelligence in Medical Imaging
}

\author{
Mohammad Majid al-Rifaie*, Ahmed Aber ${ }^{\dagger}$, Robert Sayers ${ }^{\dagger}$, Edward Choke $^{\dagger}$ and Mathew Bown ${ }^{\dagger}$ \\ *Department of Computing \\ Goldsmiths, University of London \\ London SE14 6NW, United Kingdom \\ Email: m.majid@gold.ac.uk \\ ${ }^{\dagger}$ Department of Cardiovascular Sciences \\ University of Leicester Royal Infirmary, Leicester, LE2 7LX, United Kingdom \\ Email: aa721, rs152, ec172, m.bown@le.ac.uk
}

\begin{abstract}
This paper introduces a novel approach in using a swarm intelligence algorithm - Stochastic Diffusion Search - in medical imaging. After summarising the results of some previous work - showing how the algorithm assists the identification of metastasis in bone scans and microcalcifications on the mammographs - for the first time, the use of the algorithm in assessing the CT images of aorta is demonstrated along with its performance in detecting the nasogastric tube in chest $X$-ray. The swarm intelligence algorithm presented in this paper is adapted to address these particular tasks and its functionality is investigated by running the swarms on sample $\mathrm{CT}$ images and $\mathrm{X}$-rays whose status have been determined by two senior radiologists.
\end{abstract}

\section{INTRODUCTION}

Computer aided diagnosis (CAD) is an emerging field in medicine. The technique introduced in this paper can help radiologists examine the image in greater depth and has the potential to help doctors from different medical disciplines to interpret medical imaging with greater confidence. Furthermore $\mathrm{CAD}$ is a promising learning tool for both medical students and junior doctors to develop basic diagnostic skills. This paper presents a new CAD approach in which a swarm intelligence algorithm - Stochastic Diffusion Search (SDS) [1] - is applied to four medical imaging modalities.

Understanding the basics behind the behaviour of the swarm intelligence algorithm and its connection to nature is vital. Communication - social interaction or information exchange - observed in social insects is important in all swarm intelligence algorithms, including Stochastic Diffusion Search (SDS), which mimics the recruitment behaviour of one species of ants, Leptothorax acervorum.

There are different forms of recruitment in social insects: it may take the form of local or global, one-to-one or oneto-many, and stochastic or deterministic mode. The nature of information exchange also varies in different environments and with different types of social insects. Sometimes, the information exchange is more complex where, for example, it might carry data about the direction, suitability of the target and the distance; sometimes the information sharing is simply a stimulation forcing a certain triggered action. What all these recruitment and information exchange strategies have in common is distributing useful information in their community.

This paper starts by describing the standard Stochastic Diffusion Search, followed by an introduction to bone scintigraphy, explaining metastatic disease and a brief explanation on how to detect metastasis in bone scans. Next, a brief summary of X-ray mammography and its use is presented, emphasising on mammographic film reading as a particularly demanding visual task, which could be facilitated using the technique presented in this paper.

This is followed by an introduction to aortic aneurysm disease and the factors used to assess the disease and help plan an appropriate intervention. The swarms could provide the radiologist with extra assistance in identifying the anatomy in greater detail. Then, a brief explanation is also given on nasogastric (NG) tube and its use to provide an effective mechanism through which short-term nutrition can be given to critically ill patients.

One of the serious complications of NG tube placement is caused by inadvertent malpositioning. This can lead to severe harm or death if the patient is fed through it. This could possibly be prevented by deploying the swarm intelligence technique, providing extra information about the location of the tip of the tube, avoiding fatal human error.

After presenting the above-mentioned areas of interest, a detailed introduction is given on the swarm intelligence algorithm adapted for the purpose of this research. The results are then reported for each of the areas stated earlier and an exemplar statistical analysis is given demonstrating the performance of the approach.

While the paper aims to demonstrate a novel way of addressing some of the presented medical imaging scenarios, the authors keep themselves away from the claim that the presented algorithm is an optimal solution to all medical imaging problems.

\section{A. Previous Work and Summary of Current Research}

In the initial work [2], the goal was to visualise the swarms behaviour when presented with a two dimensional canvas (e.g. bone scan). This work was well received as a potential educational tool for doctors in training and medical students. This led to the extension of the research in [3], [4] where the application of this swarm intelligence technique on bone scan was introduced in further details in different venues for researchers with medical and computer backgrounds. Later in [5], the statistical and mathematical models were presented for bone scans, and the application of the technique was extended to mammography. 
In this work, we attempt to present a unifying and generalised framework of the potential of the algorithm, showing its capability in addressing various issues related to different scans and task; after summarising the results of our previous work, two novel applications of the algorithm are introduced:

- The first one is a two-phase process in which initially the swarms identify the location of the aorta within the CT scan. The swarms then set off to detect and highlight any possible calcifications around the aorta with the goal of assisting the radiologists to determine the extent of the calcification. This information can help determine the best possible management of the disease. This task is particularly distinctive from the previous work, as a novel way of guiding the behaviour of the swarms is introduced, increasing their flexibility and functionality.

- The second application introduced in this work is the identification of the tip of the Nasogastric tube in chest $\mathrm{X}$-rays. The significance of the accurate localisation of the tip of the tube is explained with details in this work.

It is vital to note that the presented approach does not attempt to replace the experts' eyes of radiologists, however in all of the above-mentioned four applications it provides the clinicians with a valuable adjunct to aid with the diagnosis as well as the management of patient. This method of interpreting images can also be used as an educational tool for doctors in training and medical students.

\section{Stochastic Diffusion SEARCH}

This section introduces Stochastic Diffusion Search (SDS) [1] - a swarm intelligence algorithm - whose performance is based on simple interaction of agents.

The SDS algorithm commences a search or optimisation by initialising its population and then iterating through two phases (see Algorithm 1)

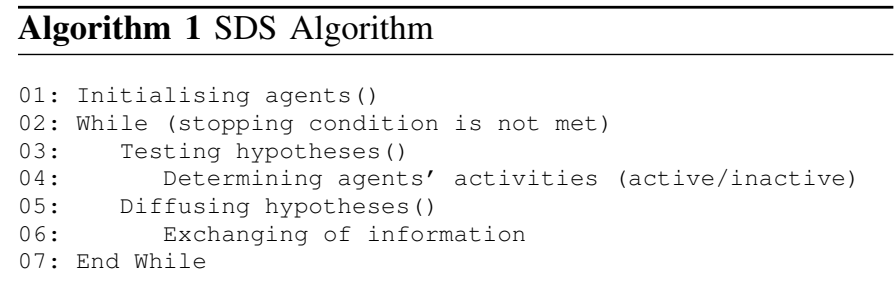

In the test phase, SDS checks whether the agent hypothesis is successful or not by performing a hypothesis evaluation which returns a boolean value. Later in the iteration, contingent on the precise recruitment strategy employed (in the diffusion phase), successful hypotheses diffuse across the population and in this way information on potentially good solutions spreads throughout the entire population of agents. In other words, each agent recruits another agent for interaction and potential communication of hypothesis. This algorithm has been used alongside other swarm intelligence algorithms in several research topics including numerical optimisation and clustering.

\section{A. Standard SDS and Passive Recruitment}

In standard SDS (which is used in this paper), passive recruitment mode is employed. In this mode, if the agent is inactive, a second agent is randomly selected for diffusion; if the second agent is active, its hypothesis is communicated (diffused) to the inactive one. Otherwise there is no flow of information between agents; instead a completely new hypothesis is generated for the first inactive agent at random (see Algorithm 2). Therefore, recruitment is not the responsibility of the active agents. In this work, activity of each agent is determined when its fitness is compared against a random agent (which is different from the selecting one); if the selecting agent has a better fitness (smaller value in minimisation problems) than the randomly selected agent, it will be flagged as active, otherwise inactive. Higher rate of inactivity boosts exploration, whereas a lower rate biases the performance towards exploitation.

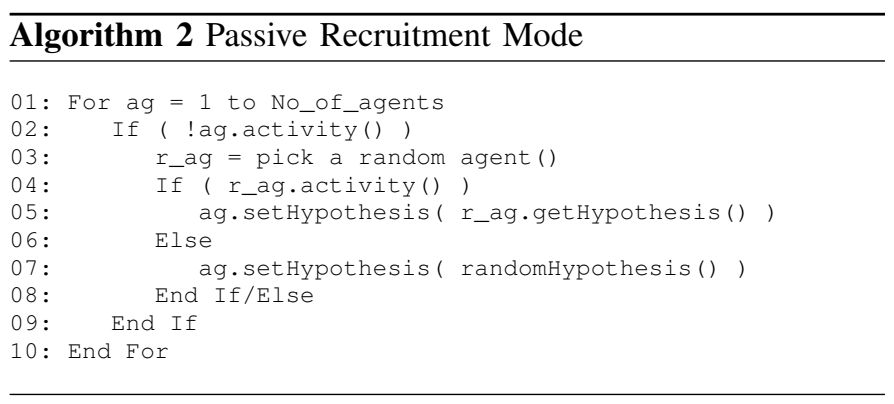

\section{BONE SCINTIGRAPHY}

Bone scan or Bone scintigraphy is one of the most frequently performed of all radionuclide procedures. Radionuclide bone imaging is quick, relatively inexpensive, widely available, exquisitely sensitive and is invaluable in the diagnostic evaluation of numerous pathologic conditions. Although protocols vary among institutions, imaging is typically performed 2-6 hours after intravenous administration of technetium-99m-labeled diphosphonates. The delay between injection and imaging allows clearance of the radiotracer from the soft tissues, resulting in a higher target-to-background ratio and improved visualization of bone. The degree of radiotracer uptake depends primarily on two factors: blood flow and, perhaps more importantly, the rate of new bone formation [6].
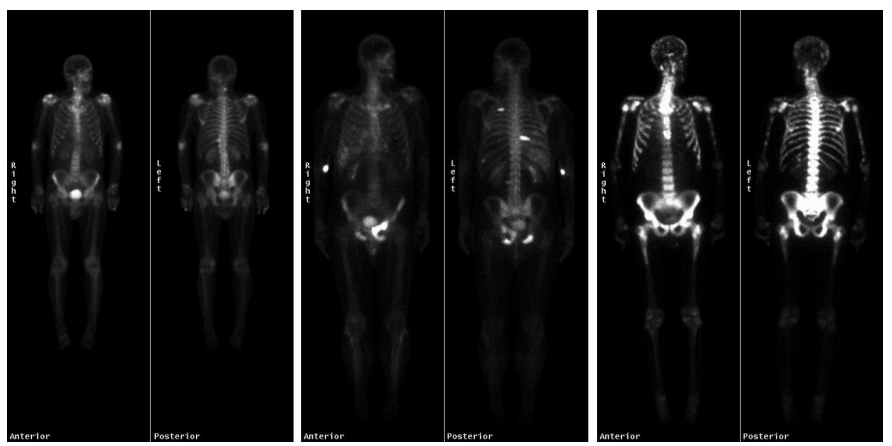

Fig. 1. Bone Scans. Typically 2-6 hours after intravenous administration of technetium-99m-labeled diphosphonates; brighter areas indicate a higher radiotracer uptake. As assessed by senior radiologists, left: Healthy; middle: partially affected; right: metastatic disease spread. 


\section{A. Normal Scintigraphic Findings}

There is symmetric distribution of activity throughout the skeletal system in healthy adults. Urinary bladder activity, faint renal activity, and minimal soft-tissue activity are also normally present (see Fig. 1 Top-left).

The accumulation of radiotracer in bone generally decreases with age. However, there are sites of persistently increased symmetric uptake, such as the acromial and coracoid processes of the scapulae, the medial ends of the clavicles, the junction of the body and manubrium of the sternum (angle of Louis), and the sacral alae. Increased radiotracer accumulation in the jaw may be due to dental disease or to malocclusion of dentures.

Symmetric areas of increased calvarial activity occurs in hyperostosis frontalis. In the neck, activity in calcified thyroid cartilage and in the apophyseal joints of the cervical vertebrae in patients with asymptomatic degenerative changes can also be seen.

\section{B. Metastatic Disease}

Metastasis is the process by which the cancer spread from the original site at which it started as a primary tumour to other tissues in the body i.e. Prostate cancer metastasising to the bone tissue.

Many if not most bone scans are performed in patients with a diagnosis of cancer, especially carcinoma of the breast, prostate gland, and lung. Radionuclide bone imaging plays an imporant part in tumor staging and management. This imaging technique is extremely sensitive for detecting skeletal abnormalities, and numerous studies have confirmed that it is considerably more sensitive than conventional radiography for this purpose [7]. About $75 \%$ of patients with malignancy and pain have abnormal bone scintigraphic findings. The usual pattern consists of increased radiotracer deposition in areas of new bone tissue formation in response to the damaging effect of cancer on the bone [7], [8]. The presence of multiple, randomly distributed areas of increased uptake of varying size, shape, and intensity are highly suggestive of bone metastases (see Fig. 1 Top-middle). Although multiple foci of increased activity may be encountered in other pathologic conditions, it is often possible to distinguish metastatic disease from other entities by analyzing the pattern of distribution of the abnormalities. Traumatic injury, in contrast to metastatic disease, generally manifests as discrete focal abnormalities of similar intensity. In older patients, osteoarthritis and degenerative changes may manifest as areas of intense activity on radionuclide bone images. These changes can be distinguished from metastatic disease by virtue of their characteristic location (e.g. knees, hands and wrists). Involvement of both sides of the joint is common in arthritis but unusual in malignant conditions [9].

When the metastatic process is diffuse, virtually all of the radiotracer is concentrated in the skeleton, with little or no activity in the soft tissues or urinary tract. The resulting pattern, which is characterized by excellent bone detail, is frequently referred to as a superscan (see Fig. 1 Top-right) [8], [9], [10].

Bone scintigraphy is a popular and important imaging modality and is likely to remain so for the foreseeable future. Although bone scintigraphy is not specific, its exquisite sensitivity makes it a useful screening procedure for many pathologic conditions, especially for the detection of prostate, breast and lung cancer metastasis.

\section{MAMMOGRAPHY}

$\mathrm{X}$-ray mammography has been shown to be effective as a method for detecting early breast cancer, but the success of mass screening depends critically on the availability of highly skilled film readers to interpret the images. The majority of film readers in the UK are consultant radiologists and in order to maintain a sufficiently high standard of interpretation, readers are required to undergo training, to keep in practice and to evaluate their performance at regular intervals [11].
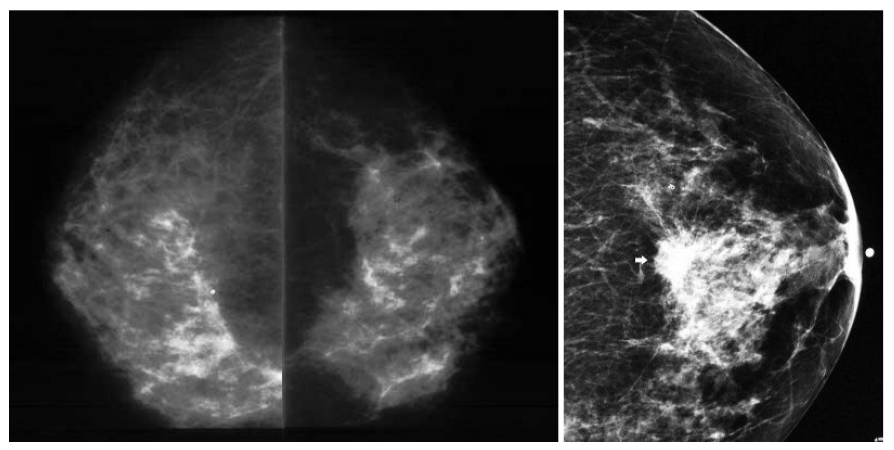

Fig. 2. Mammographs

Mammographic film reading is a particularly demanding visual task. In screening programmes, the film reader must search for extremely infrequent and often very subtle signs of cancer superimposed on complex and variable backgrounds. Early breast cancer may appear in a variety of forms: a few particles of microcalcification; a small ill-defined or speculated mass; abnormal asymmetry between right and left breast images, or subtle distortion of the underlying structure of the breast. These abnormalities vary in size, shape, structure, brightness and location and may share a great deal of similarity with normal mammographic appearances.

False negative cases, in which signs of cancer are missed by a reader, sometimes occur. Retrospective evaluation of the previous screening films of cancers detected between screening rounds (interval cancers) and screen-detected cancers show evidence of abnormality in between $16 \%$ and $27 \%$ of cases. Some of these signs are very subtle, and may have been seen by the readers but dismissed as being insignificant, but others are clear signs of malignancy [12], [13], [14]. However, different readers miss different cancers, as is evidenced by the success of double reading in which two readers independently read the films [15]. The most accurate method of interpretation is double reading with arbitration, where a third reader reviews cases about which the two readers disagree [15], [16].

In the UK particularly with the National Health Service Breast Screening Programme (NHSBSP) there is an increased demand for skilled manpower to effectively interpret mammographs and double or triple reading of the mammograph is not viable option due to the increased workload. A novel and different method of coping with this is the use of computerbased aids. 
Researchers have been developing algorithms to detect mammographic abnormalities for more than 30 years with the aim of either automating mammographic interpretation or, more realistically, providing a tool which will enhance human film-reading performance.

There are two basic approaches to the problem of detecting abnormalities in mammograms: either to search the images for specific appearances suggestive of cancer, or to characterize normal mammographic appearance to the extent that it is possible to detect anything that fails to conform to the generated model of normality.

The purpose of the current study is to apply for the first time an swarm intelligence algorithm namely Stochastic diffusion search to perform the task of identifying the microcalcifications on the mammographs.

\section{AORTIC ANEURYSM DisEASE}

The aorta is the main artery that carries the blood away from the heart to rest of the body giving rising to various branches for this purpose. Aortic aneurysm (AA) is a disease commonly found in patients above the age of 65 . It is defined as a permanent localized dilation of the aorta that has at least a 50\% increase in diameter as compared with the expected normal diameter of the aorta, which may vary according to age, sex, and body size [17].

Each year approximately 15,000 people in the United States die from a ruptured abdominal aneurysm, rendering it the $15^{\text {th }}$ leading cause of death in this country [18]. Thirty percent to $75 \%$ of patients with a ruptured Abdominal Aortic Aneurysm (AAA) die before they ever reach a hospital [19]. Even with surgery, an average $48 \%$ (95\% CI $46 \%$ to $50 \%$ ) perioperative mortality rate is associated with a ruptured AAA repair [20]. The overall mortality rate in patients with ruptured AAA ranges from $67 \%$ to $89 \%$ [19]. Therefore, detection of AAAs before rupture and elective repair can prolong survival and decrease the periprocedural complication rate.

The primary goal in Aortic Aneurysm treatment is to prolong survival through the prevention of rupture. The treatment options include the following [17]:

- Open surgical repair

- Endovascular repair (EVAR)

- Continued surveillance

Endovascular aortic aneurysm repair (EVAR) is considered a safer alternative to open surgery in selected patients. Its feasibility depends mainly on anatomic factors that represent the important predictors of success. Poor anatomic patient selection is generally associated with a higher risk for procedural complications and compromised long-term outcomes. Therefore pretreatment imaging is crucial for evaluating patient suitability for EVAR as well as appropriate planning to avoid complications mainly endoleaks. Computed tomographic angiography represents the current standard imaging used to evaluate the anatomy of aorta because it provides all the details needed for selection of patients who are suitable for EVAR.

Exclusion of the aneurysm sac is the main goal of EVAR, and clinical success is defined by the "total exclusion" of the

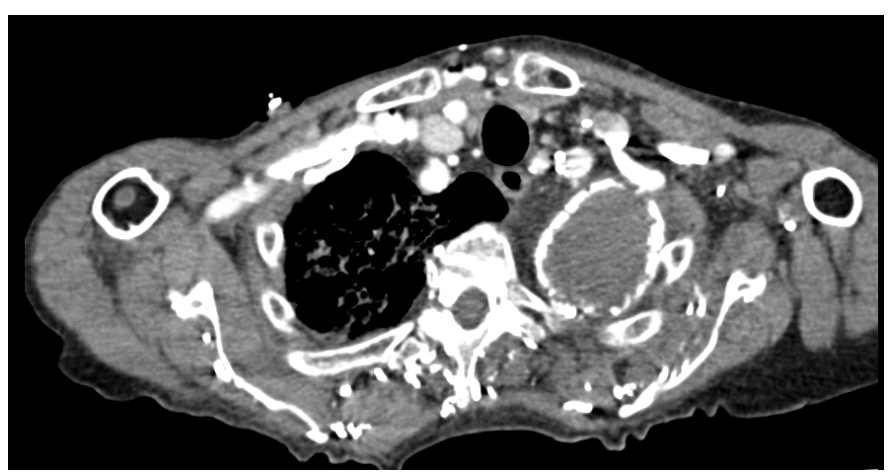

Fig. 3. A slide of CT scan showing aortic aneurysm disease

aneurysm. However, at times, failure of the stent-graft to totally exclude blood flow to the aneurysm sac may occur. As a matter of fact, endoleak is the major cause of complications, and thus failure EVAR. When an endoleak occurs, it causes continued pressurisation of the aneurysm sac and may leave the patient at risk of an AAA rupture.

\section{A. Proximal neck of the aneurysm}

The pre-EVAR anatomical evaluation assesses several aspects of the anatomy of the aortic aneurysm including the proximal neck of aneurysm anatomy which is the most important predictor factor for a successful EVAR. The proximal neck is the segment of aorta above the aneurysm sac. An unfavourable neck anatomy, based on its diameter, length, angulation, morphology, and presence of calcification and mural thrombus, is the most frequent cause of exclusion from EVAR [21].

1) Length: To achieve a good seal and decrease proximal migration and type I endoleak rates, a length of at least 15$\mathrm{mm}$ for infrarenal stent-graft fixation or $10-\mathrm{mm}$ for transrenal stent-graft fixation, with barbs, hooks, or uncovered suprarenal stents, is recommended [22]. Patients who have a neck shorter than to $10-\mathrm{mm}$ are unsuitable for EVAR

2) Angulation: A severe neck angulation $\left(>60^{\circ}\right)$ seems to be associated with a potentially higher risk of adverse events, especially type I endoleaks, as reported by studies [23], [24].

3) Morphology: The morphology of the proximal neck can be defined as straight (unchanged diameters), tapered (proximal diameter superior to distal), or reverse tapered (distal diameter superior to proximal by $>3-\mathrm{mm}$ ). A reverse tapered neck and a neck bulge are associated with a higher proximal endoleak rate and thus are contraindications for EVAR [25].

4) Presence of calcification: Calcification and mural thrombus are expressed in degrees of circumference. Mural thrombus and severe and extensive calcifications that cover more than 90 of the circumference of the aortic diameter in the proximal neck are associated with a higher risk for type I endoleak and stent-graft migration, respectively [22].

Assessing the anatomy of the aortic aneurysm for appropriate intervention is a difficult task and the use of the SDS algorithm helps segment the images and quantify some of the problems such as the calcification at different levels of the aorta. In this paper by assessing the level of calcifications at 


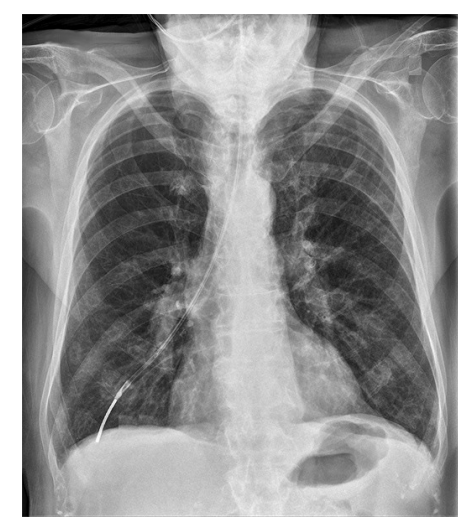

Fig. 4. Chest X-ray showing a misplaced Nasogastric tube

different parts of the proximal neck aneurysm aorta, the SDS can help identify suitable candidate for endovascular repair and potentially reduce the level of complications associated with mounting the endovascular graft in an unfavourable location.

\section{NASOgastric Tube}

Nasogastric tubes (tube inserted through the nose to the stomach) are commonly used for short-term nutrition in critically ill patients. Complications of nasogastric tubes frequently include inadvertent malpositioning and aspiration pneumonia that may cause severe harm or death. The National Patient Safety Agency (NPSA) in the United Kingdom received reports of 21 deaths and 79 cases of harm due to feeding into the lungs though misplaced NG tubes between September 2005-March 2011 [26]. The main cause of the harm in the investigated cases was the misinterpretation of the X-rays that were done to assess the position of the NG tube (see Fig. 4). X-ray assessment is usually done if the aspirate from the NG tube does not reflect the natural level of acidity of the stomach fluid content which is normally between 1-5.5 pH. It is vital to note that X-rays are used more commonly as obtaining an aspirate can be difficult in most of patients. The NPSA guidelines states that when assessing the NG position the following criteria should be strictly followed:

- The tube path follow the oesophagus/avoid the contours of the bronchi

- Assess that the tube does not bisect the carina or the bronchi (Airways)

- NG tube should cross the diaphragm in the midline

- The tip should be visible below the left hemidiaphragm

The careful interpretation of an X-ray to assess the position of an NG tube needs a trained eye. This might not be available at all times. This limitation in resources may lead to either misinterpretation by a junior doctor leading to a serious clinical incident or if there is a delay in the interpretation this can translate itself to a delay in feeding the patient for several hours. The SDS can help provide a tool to avoid the above scenarios by detecting the tip of the NG tube.

\begin{tabular}{|c|c|c|}
\hline 0 & 0 & 0 \\
\hline 0 & $x$ & 0 \\
\hline 0 & 0 & 0 \\
\hline
\end{tabular}

Fig. 5. Agent's neighbours in test phase. The symbol $x$ represents the position of the agent and the o's represent the neighbours used during the test phase. As shown in the figure, the radius of the test is set to 1 .

\section{Applying Stochastic Diffusion SEARCH}

In this paper, we are presenting a unique approach by deploying SDS to use in assessing medical images. This approach demonstrates a promising ability to undertake this task with similar level of sensitivity. Each scan used in this paper is processed by the SDS agents which are responsible for locating the desired areas.

The reproducibility and the accuracy of the SDS algorithm can be utilised in developing a standardised system to help interpreting medical images and prevent operator errors and discrepancies. This type of technologies can be employed as an adjunct to help radiologists assess the various types of images making the diagnosis more thorough and less time consuming. Additionally this technique can be effectively used to develop programs for teaching and training medical students and junior doctors.

\section{A. Algorithm Procedure}

SDS is a population based stochastic algorithm, adapted here to search for areas of metastasis or calcifications in the feasible solution space. The hypothesis vectors of the population are defined as follows:

$$
x_{i}^{g}=\left[x_{i, 1}^{g}, \ldots, x_{i, D}^{g}\right], i=1,2, \ldots, N P
$$

where $g$ is the current iteration, $D$ is the dimension of the problem space $(D=2)$ and $N P$ is the population size. In the first generation, (when $g=0$ ), the $i^{\text {th }}$ vector's $j^{\text {th }}$ component could be initialised as:

$$
x_{i, j}^{0}=x_{\min , j}+r\left(x_{\max , j}-x_{m i n, j}\right)
$$

where $r$ is a random number drawn from a uniform distribution on the unit interval $U(0,1)$, and $x_{\min }, x_{\max }$ are the lower and upper bounds of the $j^{t h}$ dimension, respectively. The initial status of all agents are set to false. In other words, each agent randomly picks a pixel from the image of the scan.

During the test phase of SDS algorithm, each agent's status should be determined. The method used here to set the activity of the agents is to find the average of the colour intensity ${ }^{1}$ (avgIn) of each agent and its neighbours (see Fig. 5). If avgIn is within a specific range (problem dependent), the agent is flagged active, otherwise inactive.

During the diffusion phase, each inactive agent randomly selects another agent from the population; if the selected agent is active, the selecting agent adopts the hypothesis (i.e. location) of the active agent and the information sharing takes place. The strategy used for information sharing is to randomly

\footnotetext{
1 Colour intensity $($ In $)$ signifies the brightness of pixels, $0 \leq \operatorname{In} \leq 255$.
} 
pick an area surrounding the active agent (see Fig. 6). Active agents also check their position by continuously picking a random pixel in the neighbourhood; this way, an area which does not have a good enough potential is discarded from one iteration (i.e. cycle of test and diffusion phases) to the next.

List of adjustable parameters for each experiments:

- $\quad$ Population size $(S)$

- Agent's activity which is determined using the value of average intensity avgIn (e.g. if avgIn $>\alpha$ the agent is set active, otherwise inactive). The value of $\alpha$ is also problem-dependent and it could be adjusted to increase or decrease the sensitivity of the system.

- Diffusion radius $(d R a d)$

- Number of iterations (Itr)

\begin{tabular}{|c|c|c|c|c|}
\hline$\circ$ & $\circ$ & $\circ$ & $\circ$ & 0 \\
\hline$\circ$ & $\circ$ & $\circ$ & $\circ$ & $\circ$ \\
\hline$\circ$ & $\circ$ & $x$ & $\circ$ & 0 \\
\hline$\circ$ & $\circ$ & $\circ$ & $\circ$ & 0 \\
\hline$\circ$ & $\circ$ & $\circ$ & $\circ$ & 0 \\
\hline
\end{tabular}

(a)

\begin{tabular}{|c|c|c|c|c|c|c|}
\hline$\circ$ & $\circ$ & $\circ$ & $\circ$ & $\circ$ & $\circ$ & 0 \\
\hline$\circ$ & $\circ$ & $\circ$ & $\circ$ & $\circ$ & $\circ$ & $\circ$ \\
\hline$\circ$ & $\circ$ & $\circ$ & $\circ$ & $\circ$ & $\circ$ & $\circ$ \\
\hline$\circ$ & $\circ$ & $\circ$ & $x$ & $\circ$ & $\circ$ & $\circ$ \\
\hline$\circ$ & $\circ$ & $\circ$ & $\circ$ & $\circ$ & $\circ$ & $\circ$ \\
\hline$\circ$ & $\circ$ & $\circ$ & $\circ$ & $\circ$ & $\circ$ & $\circ$ \\
\hline$\circ$ & $\circ$ & $\circ$ & $\circ$ & $\circ$ & $\circ$ & $\circ$ \\
\hline
\end{tabular}

(b)

Fig. 6. Diffusion area for (a) bone scans, mammographs and NG-tube; (b) AAA. The symbol $x$ represents the position of the active agent and the o's represent the accessible places during the diffusion phase. The reason behind the larger area considered for (b) is the greater radius of the area of interest in AAA (see Fig. 11).

\section{B. Bone Scans}

This section presents the results focusing on bone scans; however the methods used are extendible to other scans referred to in this work.

Below are the adjustable parameters that are determined depending on the problem in hand (i.e. identifying metastasis in bone scan) and the machine used to generate the scans. In this experiment, they are defined as:

- $\quad \mathrm{S}=10,000$

- $\quad \alpha=180$.

- $\quad \mathrm{dRad}=2$

- $\quad \operatorname{Itr}=10$

As shown in Fig. 1, areas with higher potential of metastasis are identified. In bone scans, other than urinary bladder activity, faint renal activity, and minimal soft-tissue activity which are normally present in the scan (Fig. 1 Bottomleft), the existence of multiple, randomly distributed areas
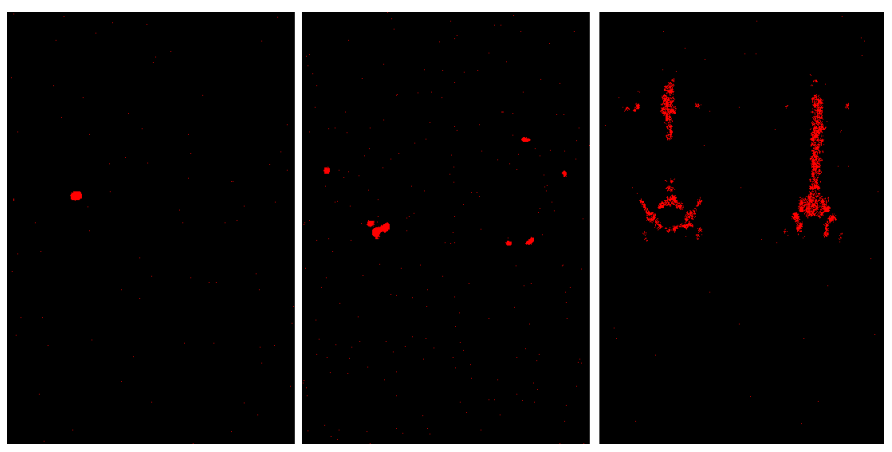

Fig. 7. The bone scans are processed using Stochastic Diffusion Search algorithm. Left: Healthy; middle: partially affected; right: metastatic disease spread.

of increased uptake of varying size, shape, and intensity are highly suggestive of bone metastases (Fig. 1 Bottom-middle). Additionally as stated before, when the metastatic process is distributed, almost all of the radiotracer congregates in the skeleton, with little or no activity in the soft tissues or urinary tract (see Fig. 1 Bottom-right).

As shown in Fig. 1 and 2, areas with higher potential of metastasis and calcifications are identified. In bone scans, other than urinary bladder activity, faint renal activity, and minimal soft-tissue activity which are normally present in the scan (Fig. 1 Bottom-left), the existence of multiple, randomly distributed areas of increased uptake of varying size, shape, and intensity are highly suggestive of bone metastases (Fig. 1 Bottom-middle). Additionally as stated before, when the metastatic process is distributed, almost all of the radiotracer congregates in the skeleton, with little or no activity in the soft tissues or urinary tract (see Fig. 1 Bottom-right).

In order to visually present the technique used, Fig. 8 illustrates how agents congregate over the areas of interest over time (i.e. iterations) when fed with the scans as inputs of the algorithm. As the figure shows, successful agents diffuse their positions across the population and this way, information on potentially good solutions spreads throughout the entire population of agents. This process is caused through the recruitment strategy, where each agent recruits another agent for interaction and potential communication of the promising areas. Next, two models are presented to distinctively differentiate between different types of bone scans (e.g. not affected, affected and highly affected).

1) Statistical Model: Here, a statistical analysis, TukeyHSD Test [27], is performed to highlight whether there is a significant difference between the activity of the agents when processing the bone scans. Table I (a) shows the activity rate of the populations over each iteration. Three different samples are used for this analysis: Samples 1, 2 and 3 refer to the scans in Fig. 1 (left to right). Table I (b) shows that other than the first iteration where the agents are just initialised, different bone scans would result in significantly different activity rates. This could be used as an indicator, highlighting the difference between various scans and whether they are healthy, partially affected or the metastasis is spread.

2) Mathematical Model: Visualising the data produced in Table I (a) could introduce another method of determining 


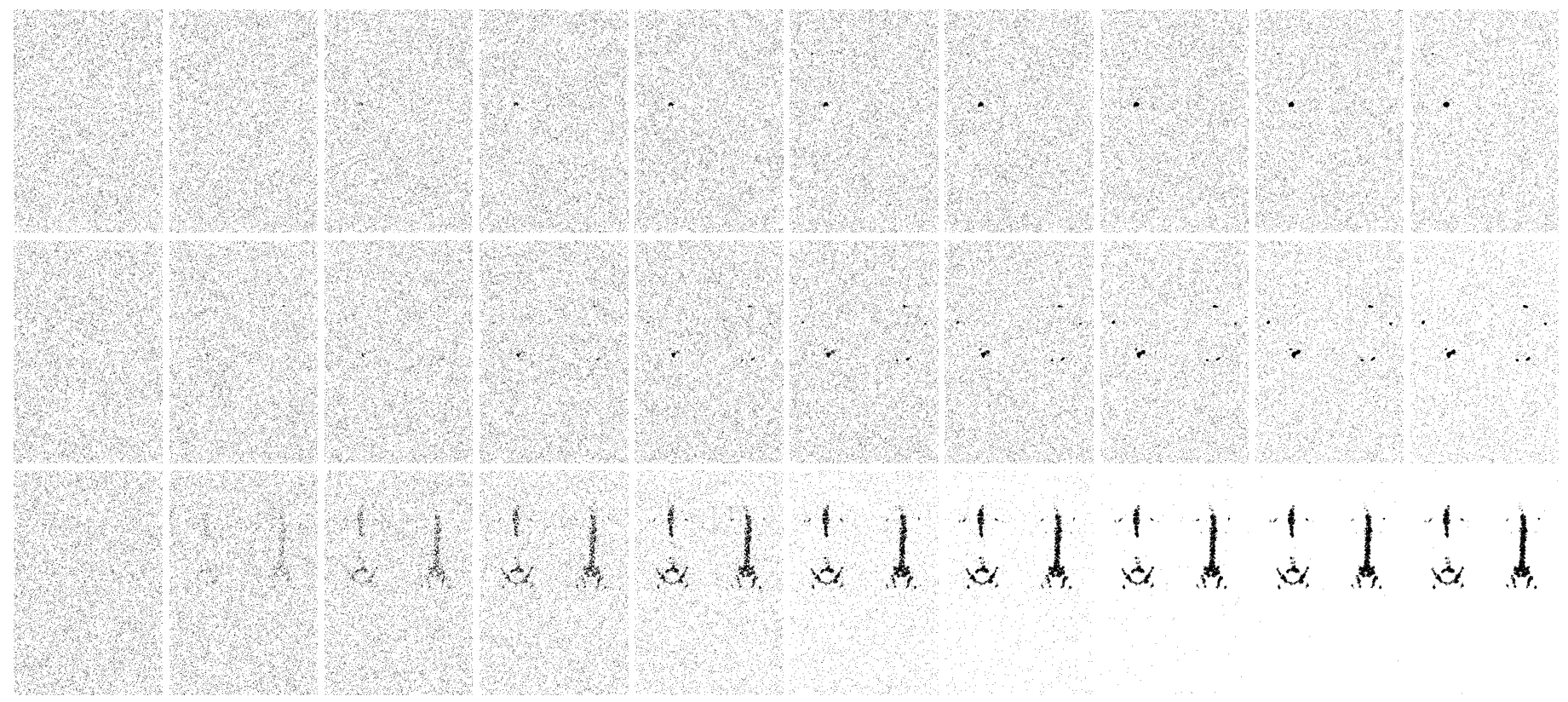

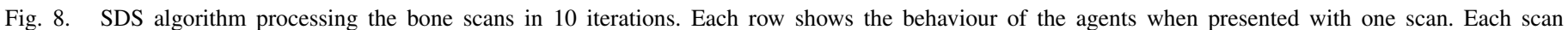

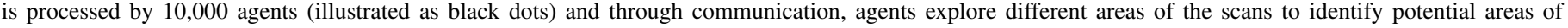
metastasis. The leftmost figures in each row show the location of the agents on the first iteration, and the rightmost ones represent the last iteration.

TABle I. Activity Status of Agents Processing Bone Scans

(a) Mean \pm standard deviation of the number of active agents in each iteration is shown (rounded to the nearest number).

\begin{tabular}{lccc} 
Itr & Sample 1 & Sample 2 & Sample 3 \\
\hline 0 & $0 \pm 0$ & $0 \pm 0$ & $0 \pm 0$ \\
1 & $5 \pm 2$ & $17 \pm 4$ & $277 \pm 16$ \\
2 & $15 \pm 4$ & $47 \pm 9$ & $763 \pm 37$ \\
3 & $33 \pm 8$ & $100 \pm 18$ & $1602 \pm 76$ \\
4 & $66 \pm 18$ & $201 \pm 31$ & $2991 \pm 137$ \\
5 & $129 \pm 33$ & $379 \pm 51$ & $4992 \pm 188$ \\
6 & $245 \pm 62$ & $697 \pm 84$ & $7260 \pm 198$ \\
7 & $461 \pm 110$ & $1250 \pm 141$ & $8947 \pm 123$ \\
8 & $852 \pm 201$ & $2201 \pm 230$ & $9583 \pm 51$ \\
9 & $1557 \pm 351$ & $3650 \pm 330$ & $9708 \pm 22$ \\
\hline
\end{tabular}

(b) Based on TukeyHSD Test, if the difference between each pair of samples is significant, the pairs are marked (o - X shows that the right sample has significantly more active agents than the left one). This test uses $95 \%$ family-wise confidence level.

The aim is to show that agents dealing with scans which have different levels of metastasis exhibit significantly different behaviour.

\begin{tabular}{cccc} 
Itr & $\mathbf{s 1 - \mathbf { s }}$ & $\mathbf{s 1 - \mathbf { s }}$ & $\mathbf{s 2}-\mathbf{s} 3$ \\
\hline 0 & - & - & - \\
1 & $\mathrm{o}-\mathrm{X}$ & $\mathrm{o}-\mathrm{X}$ & $\mathrm{O}-\mathrm{X}$ \\
2 & $\mathrm{o}-\mathrm{X}$ & $\mathrm{o}-\mathrm{X}$ & $\mathrm{O}-\mathrm{X}$ \\
3 & $\mathrm{o}-\mathrm{X}$ & $\mathrm{o}-\mathrm{X}$ & $\mathrm{O}-\mathrm{X}$ \\
4 & $\mathrm{o}-\mathrm{X}$ & $\mathrm{o}-\mathrm{X}$ & $\mathrm{O}-\mathrm{X}$ \\
5 & $\mathrm{o}-\mathrm{X}$ & $\mathrm{o}-\mathrm{X}$ & $\mathrm{o}-\mathrm{X}$ \\
6 & $\mathrm{o}-\mathrm{X}$ & $\mathrm{o}-\mathrm{X}$ & $\mathrm{o}-\mathrm{X}$ \\
7 & $\mathrm{o}-\mathrm{X}$ & $\mathrm{o}-\mathrm{X}$ & $\mathrm{o}-\mathrm{X}$ \\
8 & $\mathrm{o}-\mathrm{X}$ & $\mathrm{o}-\mathrm{X}$ & $\mathrm{o}-\mathrm{X}$ \\
9 & $\mathrm{o}-\mathrm{X}$ & $\mathrm{o}-\mathrm{X}$ & $\mathrm{o}-\mathrm{X}$ \\
\hline
\end{tabular}

which of the three broad category (healthy, partially affected or the metastasis is spread) the bone scan falls into (see Fig. 9). This model is proposed here to calculate the first and the second derivatives using the following formulas:

$$
\begin{gathered}
{f^{\prime}}_{i}^{s}=\sigma_{i}^{s}-\sigma_{i-1}^{s} \\
f_{i}^{\prime \prime s}=\sigma_{i}^{s}-2 \times \sigma_{i-1}^{s}+\sigma_{i-2}^{s}
\end{gathered}
$$

where $f^{\prime}$ and $f^{\prime \prime}$ are the first and the second derivatives respectively, $\sigma$ represents the number of active agents, $i$ is the iteration number and $s$ is the bone scan sample number, $s=\{1,2,3\}$. The value of the second derivative $\left(f^{\prime \prime}\right)$ can be used as an indicator to stop the algorithm. The rationale behind stopping the algorithm is that the activity of the agents has reached a point that allowing further process, would blur the congregation of the agents around less popular clusters. This is caused by the diffusion mechanism of the algorithm where there is higher probability of an inactive agents picking another one from within the larger clusters than the smaller ones.

Having discussed the functions of statistical and mathematical methods, the following three sections extends the use of SDS to mammographs, AAA's CT scans and NG-tube's X-ray.

\section{Mammographs}

One of the main aims of the CAD systems is to identify microcalcifications to help the radiologists make the diagnosis. Microcalcifications are sometimes difficult for the human film reader to detect because of their small size and low contrast, particularly if they are superimposed on dense glandular tissue. However, of all the signs of abnormality found on mammograms, microcalcifications are the easiest to detect automatically. Unlike small ill-defined masses, which may superficially resemble normal glandular tissue, microcalcifications have properties namely their very small size and high attenuation which differ significantly from those of normal background structures.

Adjustable parameters:

- $\quad \mathrm{S}=10,000$

- $\alpha=120$. As disucssed earlier, this experiment requires a higher level of sensitivity and thus the threshold of $\alpha$ is set lower than the previous experiment. 


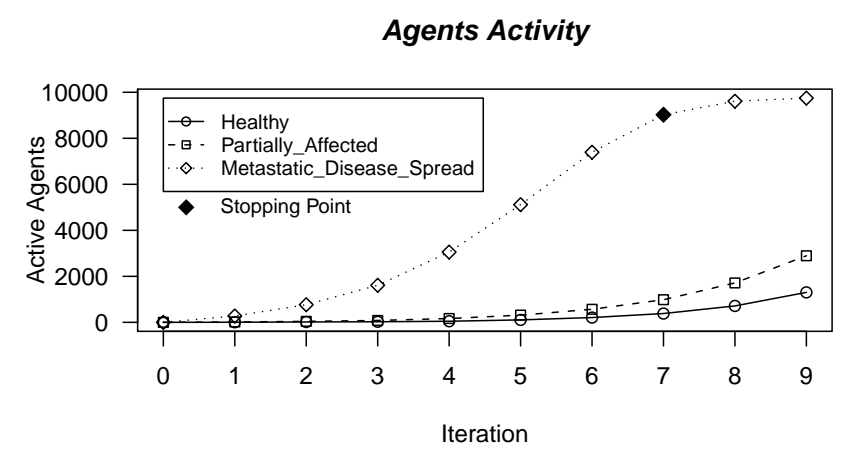

Agents Activity \& Standard Deviation

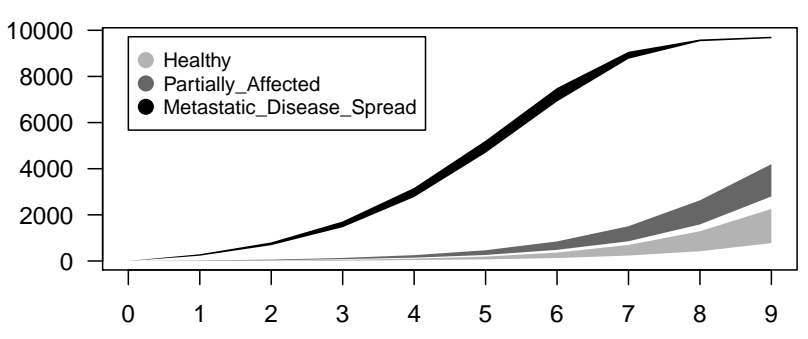

First Derivative

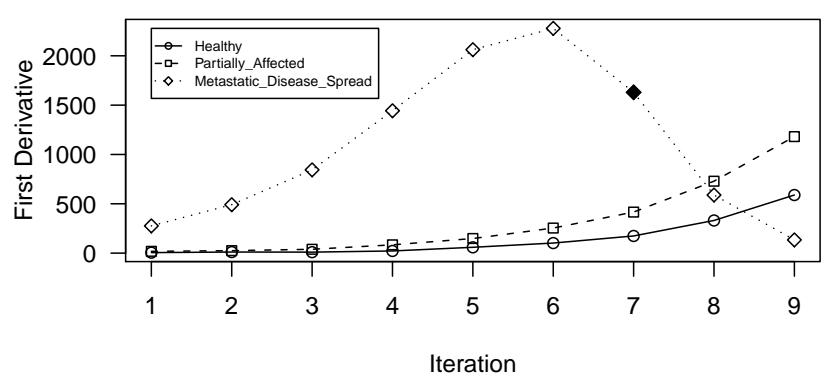

Second Derivative

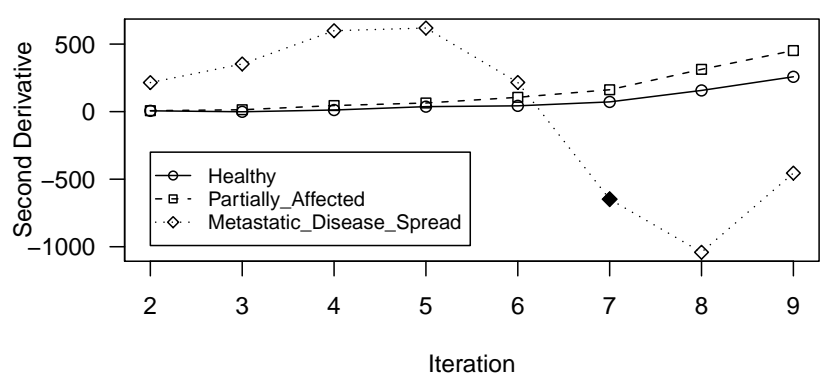

Fig. 9. Agents activity. The plots in this figure illustrate the activity of agents, the standard deviation, first and second derivatives on each iteration in the three sample bone scans. The 'stopping point' in the top plot shows the iteration number when SDS algorithm could terminate. This occurs when the value of the second derivative is negative (see Eq. 4).

- $\quad \mathrm{dRad}=2$
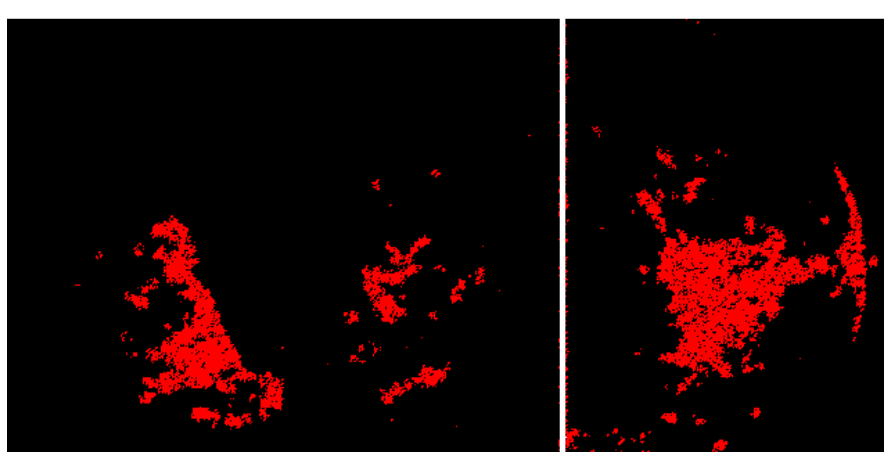

Fig. 10. Mammographs. Area with potential microcalcification particles are detected using the Stochastic Diffusion Search algorithm.

$$
\text { - } \quad \operatorname{Itr}=10
$$

Since smaller clusters are of interest in bone scan and specially mammographs, a different mechanism for determining the 'stopping point' is proposed. In this method, when $f^{\prime \prime}<0$ the algorithm reaches the stopping point. Fig. 2 shows that areas with higher potential of calcifications.

\section{Aortic Aneurysm Disease}

The SDS algorithm is utilised in this experiment to assess the proximal neck of the aneurysm and detect the level of calcification above the sac.

The agents are initialised throughout the search space (i.e. the CT image) in a two-phase mission, the first of which is to identify the location of the aorta within the scan, and the second phase highlights the existing calcification within the marked area. The adjustable parameters are empirically chosen as follows in each phase.

Phase one:

- $S=1,000$

- $\alpha=100$

- $\beta=140$

- $\quad d R a d=3$

- $\quad$ Itr $=50$

Phase Two:

- $\quad S=1,000$

- $\alpha=150$

- $\beta=255$

- $\quad d R a d=1$

- $\quad$ Itr $=100$

In the first phase, since the radius of the area of interest is in one particular area (i.e. aorta, which exists in only one place in each scan), the diffusion phase is set to explore a bigger region of the search space around the active agent and thus the diffusion radius, dRad is set to 3 units (see Fig. 6). This leads to the identification of the centre of aorta which is presented in Fig. 11 top. 
Fig. 11 middle highlights the region where potential calcifications could be spotted by the swarms (within the bigger circle in the figure); once this region is located, the second phase commences where swarms congregate over the area of interest based on the value of $\alpha<\operatorname{avgIn}<\beta$, which dictates the activity of the swarm and thus the convergence behaviour as shown in Fig. 11-bottom. As indicated above, in the second phase, the algorithm is allowed a smaller diffusion radius, $d R a d$, but a larger number of iterations in order to ensure a total convergence of the swarms to more precise areas with the required colour intensity.

Once the swarms converge, the suitability of the scan is evaluated by measuring the activity rate of the swarms; this activity is directly related to the level of calcification in the aortic wall in that particular segment of the aorta. This helps comparing different parts of the aorta to choose the best possible location with the least level of calcium in the wall of the proximal neck to position the EVAR graft.

This can help assess CT angiography images of patients awaiting the repair of the aortic aneurysm. The SDS can evaluate all the CT slides that did look at the proximal neck to identify an optimal location for deploying the graft. This can reduce the risk of the proximal type I endoleak. This type of endoleak is the most serious complication of EVAR and it is a life threatening problem. When this occur the aneurysm sac is not excluded from the circulation and it can get larger and rupture causing a vascular catastrophe with $50-75 \%$ mortality rate.

\section{E. Nasogastric Tube}

In this experiment the SDS agents are applied to identify the tip of the NG tube that is the most radiopaque part. This can help clinicians identify the distal end of the tube and assess its position to ensure that it is in the stomach and not in the lungs (see Fig. 12).

The adjustable parameters for this experiment are:

$$
\begin{array}{ll}
\text { - } & \mathrm{S}=10,000 \\
\text { - } & \alpha=240 \\
\text { - } & \beta=250 \\
\text { - } & \mathrm{dRad}=2 \\
\text { - } & \mathrm{Itr}=10
\end{array}
$$

The last three sections argue for the generalisation of the swarm intelligence technique presented in this paper. While the authors strongly keep themselves away from the any claim that the presented technique could replace human experts, the sample applications of this technique, shows the usefulness of the method and its possible potential to reduce human error and assist the identification of metastasis and micro-calcifications in various types of scans.

\section{CONCLUSION}

This paper details the promising results of the novel application of Stochastic Diffusion Search in detecting areas of metastasis in bone scans and the identification of the potential microcalcifications on the mammographs. Statistical

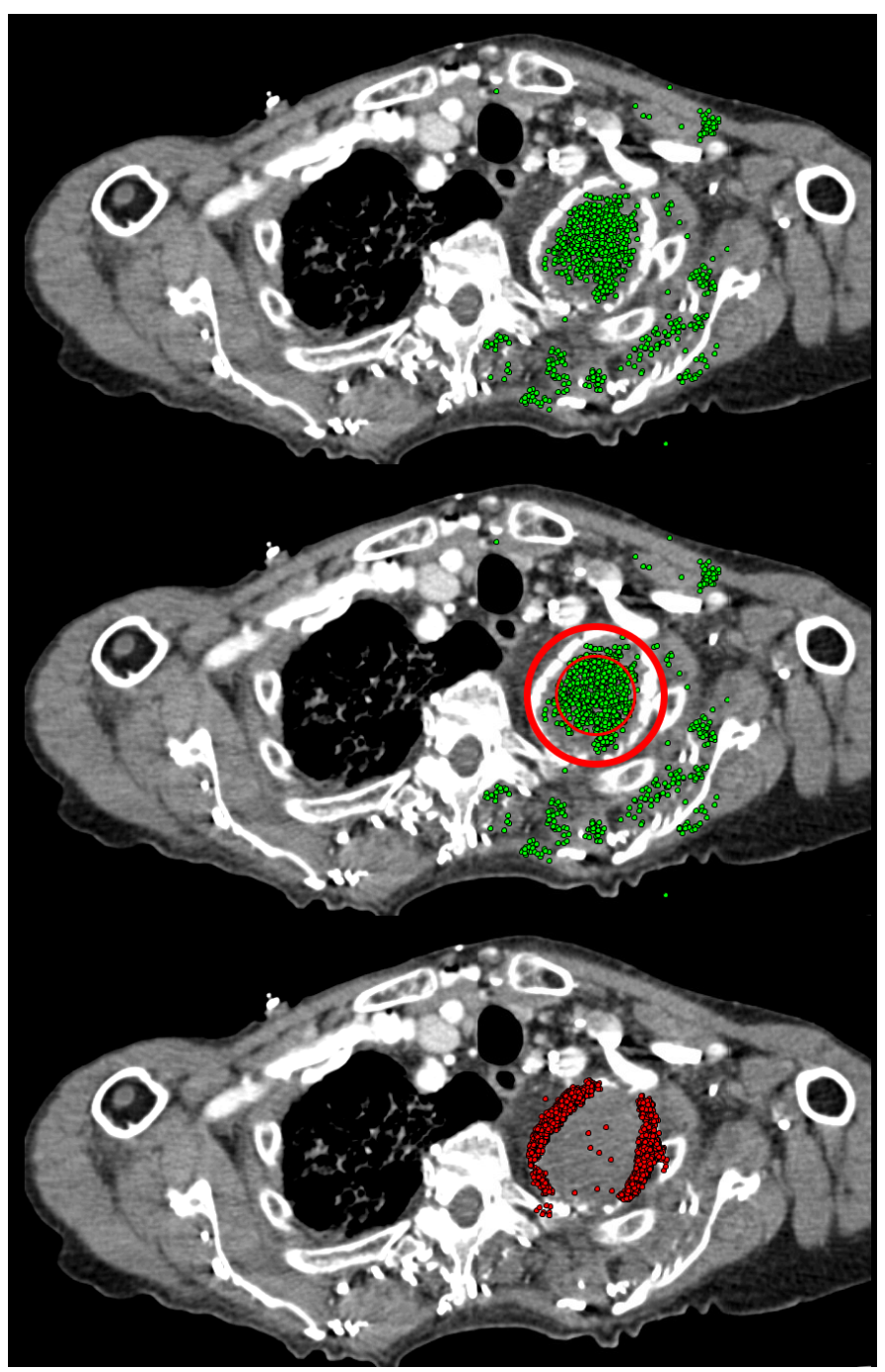

Fig. 11. Top: identifying the location of the diseased aorta within the scan; Middle: highlighting areas where potential calcifications might exist; Bottom: identification of calcification on the current slide of CT scan in order to compare with the others

and mathematical models are proposed to further investigate the behaviour of the agents in the population and the outcome demonstrates that the algorithm exhibits a statistically significant difference when applied to scans of variously affected individuals.

The swarms intelligence technique was also used in the two-phase process of the identification of aorta in the CT images, as well as calcifications in areas around the aorta; this task could lead to a more accurate localisation of the neck of the aneurysm.

Additionally, the swarms exhibited promising performance in detecting the tip of Nasogastric tube inserted through the nose to the stomach with the goal of providing short-term nutrition in critically ill patients. The identification of the tip of the tube by the swarms can in practice lead to reducing the probability of the complications of nasogastric tubes frequently caused by inadvertent malpositioning of the tube in the lungs verses stomach. 


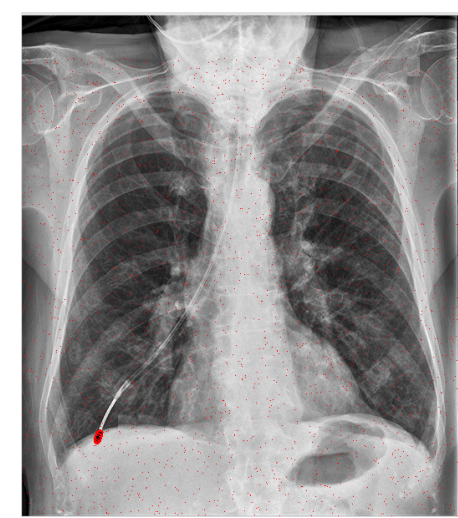

Fig. 12. Chest X-ray showing a misplaced Nasogastric tube detected by SDS

At last not least, the authors would like to emphasise that the presented technique could be effectively utilised as an adjunct to the expert's eyes of a specialist.

\section{REFERENCES}

[1] J. Bishop, "Stochastic searching networks." London, UK: Proc. 1st IEE Conf. on Artificial Neural Networks, 1989, pp. 329-331.

[2] M. M. al-Rifaie, A. Aber, and R. Raisys, "Swarming robots and possible medical applications," in International Society for the Electronic Arts (ISEA 2011), Istanbul, Turkey, 2011.

[3] M. M. al-Rifaie, A. Aber, and M. Bishop, "Swarms search for cancerous lesions: Artificial intelligence use for accurate identification of bone metastasis on bone scans," The European Federation of National Associations of Orthopaedics and Traumatology (EFORT), 13th EFORT Congress, Berlin, Germany, 2012.

[4] M. M. al-Rifaie and A. Aber, "Identifying metastasis in bone scans with stochastic diffusion search," in Information Technology in Medicine and Education (ITME). IEEE, 2012. [Online]. Available: http://dx.doi.org/10.1109/ITiME.2012.6291355

[5] M. M. al-Rifaie, A. Aber, and A. M. Oudah, "Utilising stochastic diffusion search to identify metastasis in bone scans and microcalcifications on mammographs," in Bioinformatics and Biomedicine (BIBM 2012), Multiscale Biomedical Imaging Analysis (MBIA2012). IEEE, 2012, pp. 280-287. [Online]. Available: http://dx.doi.org/10.1109/BIBMW.2012.6470317

[6] J. McAfee, R. Reba, and M. Majd, "The musculoskeletal system," Principles of Nuclear Medicine. 2nd ed. Philadelphia, PA: WB Saunders, pp. 986-1012, 1995.

[7] W. Hage, A. Aboulafia, and D. Aboulafia, "Incidence, location, and diagnostic evaluation of metastatic bone disease," Orthopedic Clinics of North America, vol. 31, no. 4, pp. 515-528, 2000.

[8] C. Bibbo, D. Patel, and J. Benevenia, "Perioperative considerations in patients with metastatic bone disease," Orthopedic Clinics of North America, vol. 31, no. 4, pp. 577-595, 2000

[9] J. Thrall and H. Ziessman, "Skeletal system," Nuclear Medicine. The Requisites, vol. 2, pp. 110-45, 2001.

[10] M. Weckesser, V. Müller-Mattheis, H. Vosberg, and H. Müller-Gärtner, "Pathogenetic differentiation of the bone superscan using bone marrow scintigraphy]." Nuklearmedizin. Nuclear medicine, vol. 37, no. 4, p. 156, 1998.

[11] C. Beam, D. Sullivan, and P. Layde, "Effect of human variability on independent double reading in screening mammography," Academic radiology, vol. 3, no. 11, pp. 891-897, 1996.

[12] J. Sumkin and D. Gur, "Computer-aided detection with screening mammography: improving performance or simply shifting the operating point?" Radiology, vol. 239, no. 3, pp. 916-918, 2006.

[13] R. Brem, J. Baum, M. Lechner, S. Kaplan, S. Souders, L. Naul, and J. Hoffmeister, "Improvement in sensitivity of screening mammography with computer-aided detection: a multiinstitutional trial," American Journal of Roentgenology, vol. 181, no. 3, pp. 687-693, 2003.
[14] A. Burgess, "On the noise variance of a digital mammography system," Medical physics, vol. 31, pp. 1987-1995, 2004

[15] E. Burnside, E. Sickles, R. Sohlich, and K. Dee, "Differential value of comparison with previous examinations in diagnostic versus screening mammography," American Journal of Roentgenology, vol. 179, no. 5, pp. 1173-1177, 2002.

[16] D. Chakraborty, "Maximum likelihood analysis of free-response receiver operating characteristic (froc) data," Medical physics, vol. 16, p. 561, 1989.

[17] K. Johnston, R. Rutherford, M. Tilson, D. Shah, L. Hollier, and J. Stanley, "Suggested standards for reporting on arterial aneurysms," Journal of vascular surgery, vol. 13, no. 3, pp. 452-458, 1991.

[18] N. C. for Health Statistics, "Deaths, percent of total deaths and death rates for the 15 leading causes of death: United states and each state, 2000," National Center for Health Statistics, 2001.

[19] K. Cassar, D. Godden, and J. Duncan, "Community mortality after ruptured abdominal aortic aneurysm is unrelated to the distance from the surgical centre," British journal of surgery, vol. 88, no. 10, pp. 1341-1343, 2001.

[20] L. Brown, J. Powell et al., "Risk factors for aneurysm rupture in patients kept under ultrasound surveillance," Annals of surgery, vol. 230, no. 3, p. 289,1999 .

[21] F. Arko, K. Filis, S. Seidel, J. Gonzalez, S. Lengle, R. Webb, J. Rhee, and C. Zarins, "How many patients with infrarenal aneurysms are candidates for endovascular repair? the northern california experience," Journal Information, vol. 11, no. 1, 2004.

[22] M. Armerding, G. Rubin, C. Beaulieu, S. Slonim, E. Olcott, S. Samuels, M. Jorgensen, C. Semba, R. Jeffrey, and M. Dake, "Aortic aneurysmal disease: Assessment of stent-graft treatmentct versus conventional angiography1," Radiology, vol. 215, no. 1, pp. 138-146, 2000.

[23] W. Sternbergh, G. Carter, J. York, M. Yoselevitz, and S. Money, "Aortic neck angulation predicts adverse outcome with endovascular abdominal aortic aneurysm repair," Journal of vascular surgery, vol. 35, no. 3, pp. 482-486, 2002.

[24] M. Robbins, B. Kritpracha, H. Beebe, F. Criado, Y. Daoud, and A. Comerota, "Suprarenal endograft fixation avoids adverse outcomes associated with aortic neck angulation," Annals of vascular surgery, vol. 19, no. 2, pp. 172-177, 2005.

[25] B. Stanley, J. Semmens, Q. Mai, M. Goodman, D. Hartley, C. Wilkinson, and M. Lawrence-Brown, "Evaluation of patient selection guidelines for endoluminal aaa repair with the zenith stent-graft: the australasian experience," Journal Information, vol. 8, no. 5, 2001.

[26] N. Guidelines, "Reducing the harm caused by misplaced nasogastric feeding tubes in adults, children and infants," NHS, 2011.

[27] R. Miller, "Simultaneous statistical inference," SPRINGER-VERLAG INC., 175 FIFTH AVE., NEW YORK, NY, 1981, 300, 1981. 